\title{
AxI-mediated activation of TBK1 drives epithelial plasticity in pancreatic cancer
}

\author{
Victoria H. Cruz, ${ }^{1}$ Emily N. Arner, ${ }^{1}$ Wenting Du, ${ }^{1}$ Alberto E. Bremauntz, ${ }^{2}$ and Rolf A. Brekken ${ }^{1,3}$ \\ 'Division of Surgical Oncology, Department of Surgery, and the Hamon Center for Therapeutic Oncology Research, \\ ${ }^{2}$ Department of Internal Medicine, and ${ }^{3}$ Department of Pharmacology, University of Texas Southwestern Medical \\ Center, Dallas, Texas, USA.
}

Pancreatic ductal adenocarcinoma (PDA) is characterized by an activating mutation in KRAS. Direct inhibition of KRAS through pharmacological means remains a challenge; however, targeting key KRAS effectors has therapeutic potential. We investigated the contribution of TANK-binding kinase 1 (TBK1), a critical downstream effector of mutant active KRAS, to PDA progression. We report that TBK1 supports the growth and metastasis of KRAS-mutant PDA by driving an epithelial plasticity program in tumor cells that enhances invasive and metastatic capacity. Further, we identify that the receptor tyrosine kinase Axl induces TBK1 activity in a Ras-RalB-dependent manner. These findings demonstrate that TBK1 is central to an Axl-driven epithelial-mesenchymal transition in KRASmutant PDA and suggest that interruption of the AxI/TBK1 signaling cascade above or below KRAS has potential therapeutic efficacy in this recalcitrant disease.

\section{Authorship note: VHC and ENA contributed equally to this work. \\ Conflict of interest: The authors have declared that no conflict of interest exists.}

Copyright: (c) 2019 American Society for Clinical Investigation

Submitted: November 9, 2018 Accepted: March 27, 2019 Published: May 2, 2019.

Reference information: /CI Insight. 2019;4(9):e126117. https://doi. org/10.1172/ji.insight.126117.

\section{Introduction}

Pancreatic ductal adenocarcinoma (PDA) is a lethal and poorly understood human malignancy with patient survival that has not improved substantially in the last 40 years (1). Each year, more than 45,000 new cases are diagnosed, and an almost equal number of patients succumb to the disease (1). The high incidence-to-death ratio is attributed to therapy resistance and to late diagnosis, at which point the tumor has metastasized (2).

Significant effort has gone into identifying critical pathways for PDA growth that can be exploited therapeutically. Understanding the biology of activating point mutations in the small GTPase KRAS, which is an early genetic event in human PDA development and is present in $90 \%$ of PDA cases (3), has been a focal point of drug development strategies. Oncogenic KRAS is the dominant driver in PDA initiation and maintenance (4); however, RAS itself has not been an amenable target for direct inhibition. As a result, developing therapeutic strategies that inhibit RAS effector signaling is attractive (5).

Although the majority of RAS effector-targeted therapies are focused on the RAF and PI3K signaling networks, there is considerable evidence supporting the less studied RALGEF/RAL effector pathway as a critical contributor to PDA growth (6-8). Though not mutated as frequently as the RAF/MEK/ERK and PI3K signaling molecules in human cancer, the RALA and RALB GTPases of the RALGEF pathway are more consistently activated than RAF or PI3K in human pancreatic tumors (6-8). The serine/threonine protein kinase TANK-binding kinase 1 (TBK1) is a major constituent of the RAL pathway and is critical to the development of RAS-driven cancers $(9,10)$. We previously reported that $T B K 1$ expression is associated with a poor prognosis in pancreatic cancer patients (stages I-II) from The Cancer Genome Atlas (11). High expression of $T B K 1$ showed a trend toward poorer overall survival in this patient cohort $(P=$ 0.07). Studies in lung cancer revealed that RALB activates TBK1, leading to the restriction of apoptosis, while having no effect on survival of nontumorigenic epithelial cells (12). Moreover, the expression of an oncogenic KRAS allele in TBK1-deficient murine embryonic fibroblasts was found to induce cell death, suggesting that TBK1 is integral for cells to tolerate transforming levels of oncogenic RAS (12). The critical contribution of RALB and TBK1 to RAS-induced lung cancer growth was corroborated in an RNAi screen of synthetic lethal partners of oncogenic KRAS, where RALB and TBK1 were identified as top hits (10).

Given the prominent activity of RalB in pancreatic cancer and the requirement of TBK1 for cells to tolerate transforming levels of oncogenic RAS, we hypothesized that TBK1 is critical to KRAS-driven pancreatic cancer growth. Here we show that TBK1 is highly expressed in $K R A S$-mutant pancreatic cancer. We found that the loss of TBK1 function in preclinical mouse models of $K R A S$-mutant PDA resulted in 
reduced tumor load and reduced metastatic events, indicating that TBK1 activity contributes directly to the aggressive properties of pancreatic cancer. Taken together, our findings highlight TBK1 inhibition as a potentially novel approach to targeting $K R A S$-mutant pancreatic cancer.

\section{Results}

TBK1 expression in pancreatic cancer. TBK1 is expressed in numerous epithelial tumors, including those of the breast, lung, and colon (10, 13-15). However, the function and activity of TBK1 in human pancreatic cancer has not been characterized extensively. We found that TBK1 was expressed and was active (phosphorylated TBK1, p-TBK1) under basal conditions in a panel of human KRAS-mutant PDA cell lines. In contrast, TBK1 was expressed but with reduced activity in normal pancreatic (nestin-expressing) ductal epithelial cells (HPNEs) (16) or KRAS-wild-type PDA cells. Mouse embryonic fibroblasts isolated from Tbk1-mutant mice $\left(T b k 1^{4 / 4}\right)$ (17) served as a negative control with no detectable expression of TBK1 or p-TBK1 (Figure 1A). Additionally, we observed higher TBK1 protein levels in spontaneous pancreatic tumors from genetically engineered mouse models (GEMMs) compared with normal pancreas samples from littermate controls (Figure 1B). Though not causative, these data suggest that TBK1 expression and activity are important in human pancreatic cancer.

$K R A S$-driven pancreatic cancer growth is disrupted by restricting TBK1 activity. To assess the contribution

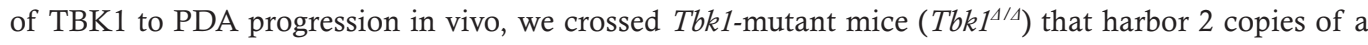
null Tbk1 allele into a GEMM of PDA. The "null" Tbk1 allele encodes a truncated TBK1 protein that is kinase inactive and expressed at low levels, thereby allowing analysis of global TBK1 loss in vivo (17). $T b k 1^{1 / 4}$ animals were crossed with $\mathrm{Kras}^{L S L-G 12 D /+} C d k n 2 a^{L o x / L o x} \mathrm{Ptfla}^{\mathrm{Cre} /+}$ (KIC) mice. KIC mice present with low-grade ductal lesions by 3 weeks of age (18-20) that progress to pancreatic adenocarcinomas such that all mice are moribund between 7 and 11 weeks of age. We hypothesized that TBK1 was critical for

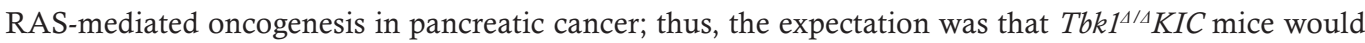
have smaller tumors and outlive $T b k 1^{+/+} K I C$ mice. In comparing tumor sizes, we observed that tumors from $T b k 1^{\Delta / \Delta}$ KIC mice were between $20 \%$ and $40 \%$ smaller than $T b k 1^{+/+} K I C$ tumors at multiple time points, yet there was no difference in overall survival between the 2 groups (Figure 2, A and B). Malnutrition resulting from loss of normal exocrine pancreas function and pancreatic enzyme insufficiency can contribute to early death in the KIC model (21). Thus, we cannot exclude the possibility that the antitumor effects of Tbk1 loss are surpassed by malnutrition induced the KIC model.

Tbk1/4KIC tumors are differentiated. To better understand TBK1-dependent mechanisms of tumor cell growth contributing to larger tumors in KIC mice, we performed gene expression analysis using RNA isolated from 8-week-old $T b k 1^{4 / 4} K I C$ and $T b k 1^{+/+} K I C$ tumors. One of the most significant and top dysregulated gene networks between $T b k 1^{\Delta / \Delta} K I C$ and $T b k 1^{+/+} K I C$ tumors identified by Ingenuity Pathway Analysis (IPA, Qiagen) was the cancer/cellular movement network. This network included a large number of genes involved in epithelial-mesenchymal transition (EMT). In comparison with $T b k 1^{+/+} K I C$ tumors, all $3 \mathrm{Tbk} 1^{1 / \Delta} \mathrm{KIC}$ tumors showed a trend of lower expression of mesenchymal genes, such as vimentin and matrix metallopeptidase 9 (MMP-9), and higher expression of epithelial genes, including claudin-3, -4 , and -10 and tissue inhibitor of metalloproteinase 3 (Figure $3 \mathrm{~A}$ ). Tbk ${ }^{+/+} \mathrm{KIC}$ and $\mathrm{Tbk} 1^{4 / 4} \mathrm{KIC}$ tumors were characterized by IHC for epithelial and mesenchymal markers (Figure 3B). Tbk $1^{4 / 4} K I C$ tumors expressed significantly higher levels of epithelial markers, such as E-cadherin, cytokeratin-19 (CK-19), and claudin-1, whereas $T b k 1^{+/+} K I C$ tumors showed significantly higher levels of mesenchymal markers, such as Slug and Zeb-1 (Figure 3). Additionally, Tbk $1^{\Delta / 4} K I C$ tumors also expressed a higher level of the mitotic marker Ki67, indicative of increased cell proliferation, a phenotype consistent with epithelial/ differentiated tumors (Figure 3). Low vimentin expression was also confirmed at the protein level by immunofluorescent staining of tumors from 8-week-old animals (Supplemental Figure 1; supplemental material available online with this article; https://doi.org/10.1172/jci.insight.126117DS1). Further, this EMT gene expression signature was consistent with Alcian blue staining of $T b k 1^{4 / \Delta} K I C$ and $T b k 1^{+/+} K I C$ tumors. Alcian blue stains mucins that are expressed by ductal epithelial cells in pancreatic intraepithelial neoplasia lesions (20). Representative images show that $T b k 1^{4 / \Delta} K I C$ tumors contain more Alcian blue-positive epithelial cells compared with less differentiated $\mathrm{Tbk1}^{+/+} \mathrm{KIC}$ tumors (Supplemental Figure 1). Furthermore, we evaluated collagen deposition, a hallmark of pancreatic cancer that promotes EMT in PDA and is upregulated in response to epithelial plasticity $(20,22) . T b k 1^{+/+} K I C$ tumors showed higher levels of fibrillar collagen than $T b k 1^{4 / 4} K I C$ tumors by trichrome histology (Supplemental Figure 1). 


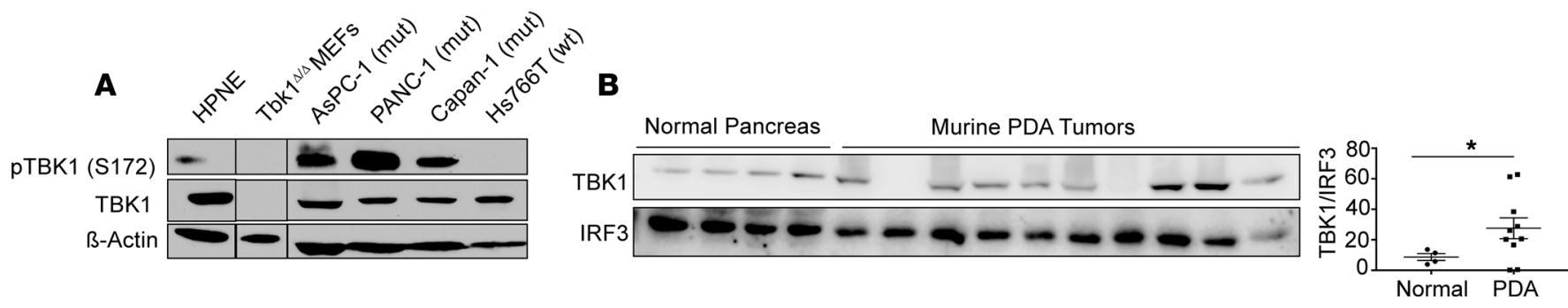

Figure 1. TBK1 is highly expressed in pancreatic cancer. (A) Total and p-TBK1 (S172) expression in wild-type KRAS (wt) or mutant active KRAS (mut) HPNE or PDA cell lines. Tbk $1^{1 / 4}$ mouse embryonic fibroblasts served as a negative control. We used $\beta$-actin as a loading control. Solid line indicates where blot was cropped; however, all samples were run on the same gel and exposed simultaneously. Western blots displayed are representative of $n>3$ repeats. (B) Total TBK1 expression in murine PDA tumors relative to normal pancreas from non-tumor-bearing littermate controls. Total IRF3 was used as a loading control. Intensity quantification is representative of mean \pm SEM. ${ }^{*} P<0.05$ by unpaired, 2 -tailed $t$ test.

To confirm the tumor cell epithelial phenotype observed in $T b k 1^{1 / 4} \mathrm{KIC}$ tumors, we isolated single-cell clones from $T b k 1^{+/+} K I C$ and $T b k 1^{1 / 4} K I C$ tumors. In total, 3 cell lines per genotype were generated, each from individual tumors. In accordance with gene expression data from $T b k 1^{+/+} K I C$ tumors, each cell line isolated showed evidence of EMT with an elongated, spindle-like cell shape, a characteristic often associated with mesenchymal cells. Moreover, cell lines from $T b k 1^{1 / 4} K I C$ tumors exhibited a "cobblestone" morphology, a feature consistent with epithelial cells. These differences in morphology were observed in organotypic culture after the cells were plated on a mixed layer of collagen and Matrigel and fixed and stained to highlight the F-actin (Figure 4A). Evaluation of EMT-related markers revealed higher expression of epithelial proteins zona occludens 1 (ZO-1) and E-cadherin and lower expression of the mesenchymal proteins vimentin, Slug, and Snail in $T b k 1^{1 / 4} K I C$ cell lines (Figure 4B). Because these generated cell lines are made from single clones from individual animals, some heterogeneity was observed. This is to be expected because pancreatic cancer is known to be a heterogeneous disease. These results illustrate a unique epithelial signature in Tbk ${ }^{1 / 4} \mathrm{KIC}$ tumor cells and have implications for functional differences in tumor cell motility.

Tbk $1^{1 / 4} \mathrm{KIC}$ tumor cells are less migratory and less invasive. Epithelial plasticity changes commonly correspond with alterations in tumor cell motility and invasiveness (23). Given that Tbk $1^{1 / 4} \mathrm{KIC}$ tumors and cell lines are less mesenchymal in gene expression and morphology, we hypothesized that functional TBK1 is important for tumor cell migration. To compare motility and invasiveness between $T b k 1^{+/+} K I C$ and $T b k 1^{1 / 4} \mathrm{KIC}$ tumor cell lines, we performed a series of wound healing and Transwell invasion assays. Despite the fact that $T b k 1^{1 /}$ ${ }^{4} \mathrm{KIC}$ cell lines proliferate more quickly in culture (Supplemental Figure 2A), they did not migrate as well as $\mathrm{Tbk1}^{+/+} \mathrm{KIC}$ cells (Supplemental Figure 2B). ECM-coated Transwell migration assays also revealed a $20 \%$ to

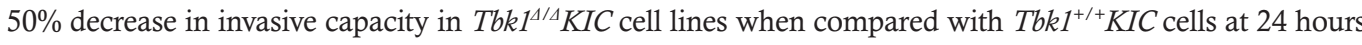
(Supplemental Figure 2C). These results further highlight the reduced migratory ability of $T b k 1^{1 / 4} \mathrm{KIC}$ cells.

Evaluation of Tbk1 loss on pancreatic cancer metastases. Next, we asked whether the reduction in tumor cell motility with kinase-dead Tbk1 translated to fewer metastases in vivo. The KIC mouse model of PDA is an aggressive model with an average life span of approximately 10 weeks (18-20). As such, these mice rarely develop gross metastases, making this model less than ideal for comparing metastatic burden. However, it's worth mentioning that livers from $\mathrm{Tbk}^{+/+}(n=14)$ and $\mathrm{Tbk} 1^{1 / 4}(n=12) \mathrm{KIC}$ mice were examined for micrometastases using histology and quantitative PCR (Supplemental Figure 3, A and B). Lesions were identified in 6 livers from $\mathrm{Tbk}^{+/+}$animals, but no lesions were found in livers from Tbk1-mutant mice. To more robustly study the effect of Tbk1 loss on metastatic potential, we used 2 animal models. First, we exploited an experimental metastasis model where $T b k 1^{+/+}$or $T b k 1^{1 / 4} \mathrm{KIC}$ cell lines were injected intravenously (i.v.) into NOD/SCID mice and lung colonization was determined after 12 days. Tbk1-mutant cells were less efficient at forming lung lesions as evidenced by gross lesion formation, by H\&E, and by lung weight (Figure 5, A-D).

Although the experimental metastasis assay results were striking, the effect of Tbk1 loss on spon-

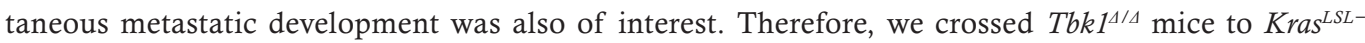
${ }^{G 12 D /+} \mathrm{LSL}-\operatorname{Trp} 53^{\mathrm{LSL}-\mathrm{R} 172 \mathrm{H} /+} \mathrm{Ptf1} \mathrm{a}^{\mathrm{Cre} /+}(\mathrm{KPC})$ animals. The KPC model differs from the KIC model in that it contains a dominant-negative p53 point mutation instead of loss of the tumor suppressor Cdkn2a (24). $K P C$ mice have a longer median survival (5 months), allowing more time for tumor cells to metastasize $(24,25)$. Although not statistically significant, $T b k 1^{4 / 4} K P C$ mice live 1 month longer than $T b k 1^{+/+} K P C$ mice, shifting the median survival from 5 months to 6 months $(P=0.15)$ (Figure 6A). Primary tumor 

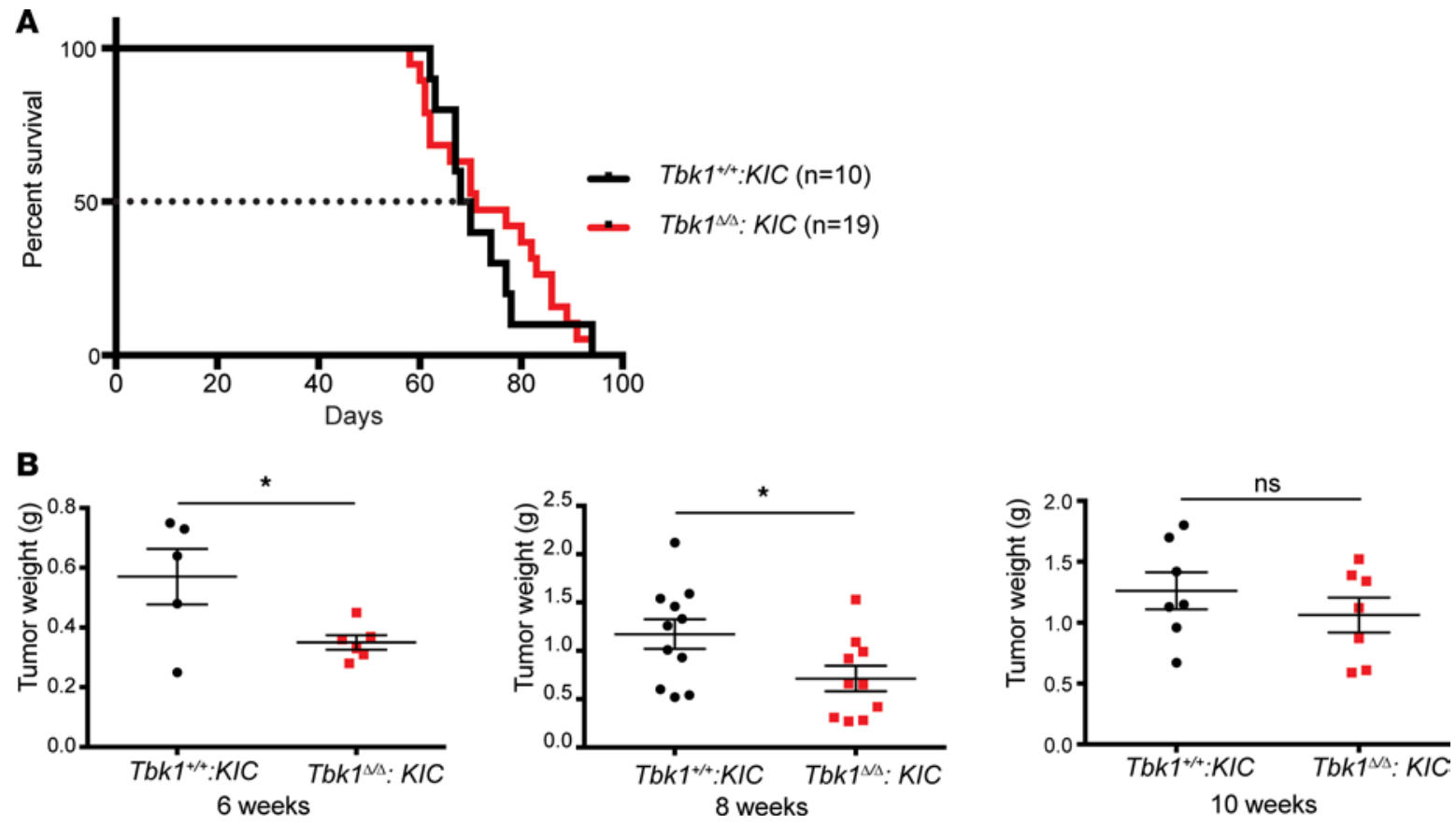

Figure 2. TBK1 promotes PDA. (A) Kaplan-Meier survival curve of Tbk $1^{1 / 4} K I C$ and $T b k 1^{+/+} K I C$ mice. A log-rank Mantel-Cox test was used for survival comparison. (B) Endpoint tumor weights at 6, 8, and 10 weeks in $T b k 1^{1 / 4} \mathrm{KIC}$ and $T b k 1^{+/+} \mathrm{KIC}$ mice. Results are representative of mean $\pm \mathrm{SEM}$. ${ }^{*} P<0.05$ by unpaired, 2-tailed $t$ test. ns, not significant.

burden was significantly reduced in $T b k 1^{1 / 4} \mathrm{KPC}$ animals relative to $\mathrm{Tbk1^{+/+ }} \mathrm{KPC}$ animals (Figure 6B). Although we did not investigate the immune landscape of the PDA GEMMs, we did find via gene expression analysis that $T b k 1^{\Delta / A} K I C$ tumors displayed a higher expression of a number of proinflammatory genes relative to $T b k 1^{+/+} K I C$ tumors (Supplemental Figure 4). The elevated proinflammatory gene expression in $T b k 1^{1 / 4} \mathrm{KIC}$ tumors could be indicative of a heightened antitumor immune response to some degree, resulting in smaller tumors.

Like the KIC model, Tbk $1^{1 / 4} K P C$ tumors showed significantly greater levels of E-cadherin, whereas $T b k 1^{+/+} K P C$ tumors showed significantly higher expression of mesenchymal markers, such as vimentin and Zeb-1, indicating $T b k 1^{1 / 4} \mathrm{KPC}$ tumors are more epithelial relative to $\mathrm{Tbk}^{+/ /+} \mathrm{KPC}$ tumors (Figure $6 \mathrm{C}$ ). Liver and lung metastases were evaluated in gross (Figure 6D) and by H\&E, Alcian blue, and CK-19 immunohistochemical staining (Figure 6E). As expected, approximately $40 \%$ of $\mathrm{Tbk}^{+/+} \mathrm{KPC}$ mice were positive for metastasis, but strikingly, no metastatic lesions were detected in $T b k 1^{1 / 4} \mathrm{KPC}$ mice. To confirm the EMT phenotype of the tumor cells, we again isolated single-cell clones from individual $T b k 1^{+/+} K P C$ and $T b k 1^{1 / 4} \mathrm{KPC}$ tumors. Consistent with gene expression data from $T b k 1^{+/+} K I C$ tumor cell lines, evaluation of EMT-related markers revealed higher expression of the epithelial protein ZO-1 and lower expression of the mesenchymal proteins Slug and Zeb-1 in $T b k 1^{1 / 4} K P C$ cell lines (Supplemental Figure 5). These data suggest loss of functional Tbk1 in the KPC GEMM restricts tumor cell metastases.

Reexpression of Tbk1 in Tbk $1^{1 / 4} \mathrm{KIC}$ cells. To confirm that TBK1 promotes pancreatic tumor cell motility, we stably reexpressed full-length human $T B K 1$ by lentiviral infection in $T b k 1^{1 / 4} K I C$ tumor cells and assayed them for invasive and migratory activity. Reexpression of $T B K 1$ in $T b k 1^{1 / 4} K I C$ tumor cells was

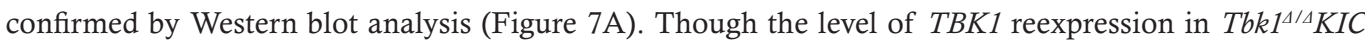
tumor cell lines was substantially lower than endogenous TBK1 levels in $T b k 1^{+/+} K I C$ cell lines, we did detect a rescue of the mesenchymal morphology in $T b k 1^{4 / 4} \mathrm{KIC}$ cells infected with TBK1-expressing lentivirus ( $\mathrm{pCDH}-\mathrm{TBK} 1$ ) compared with empty vector-expressing (pCDH-empty vector) cells (Figure 7B and Supplemental Figure 6). Further, Tbk $1^{4 / 4} \mathrm{KIC}$ cell lines rescued with $\mathrm{pCDH}-\mathrm{TBK} 1$ formed 2-3 times as many lung tumor nodules, resulting in greater diseased lung burden than cells infected with $\mathrm{pCDH}-$ empty vector after i.v. injection (Figure 7, C-E). These results demonstrate that Tbk1 loss is responsible for the migratory and invasive deficiency in $T b k 1^{1 / 4} K I C$ cells and highlight a potentially novel function for TBK1 in promoting a migratory program in tumor cells. 
A

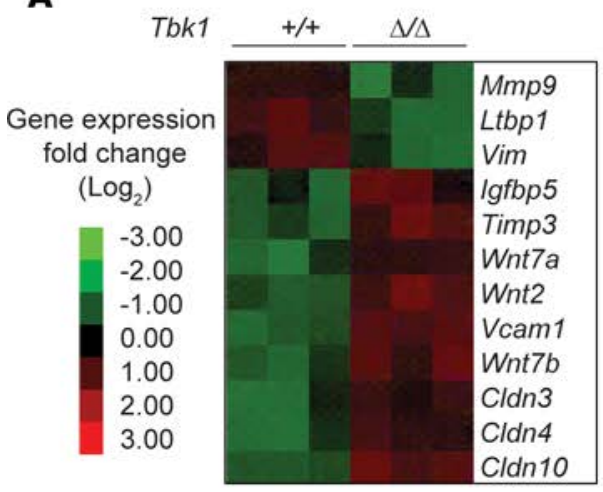

B

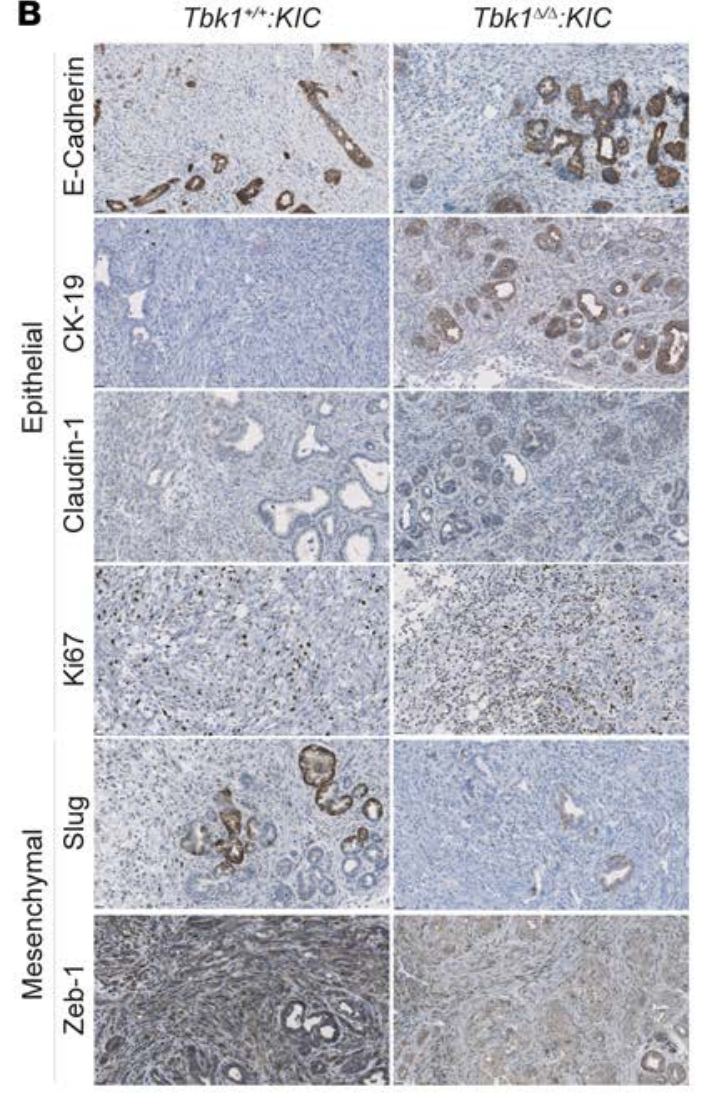

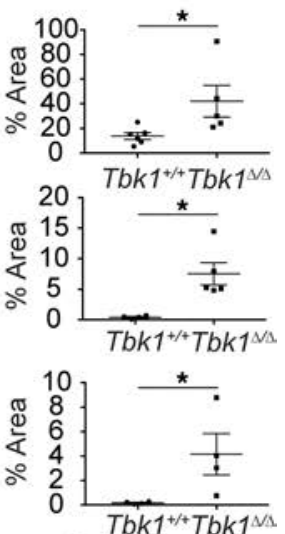
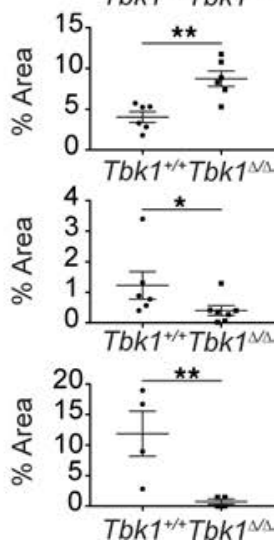

Figure 3. Loss of Tbk1 results in tumor cell epithelial differentiation. (A) Heatmap representing gene expression fold change (log lof $_{2}$ EMT-related genes from $T b k 1^{+/+} K I C$ and $T b k 1^{1 / 4} K I C$ tumors. Color key indicates gene expression fold change; $n=3$ tumors/genotype; $P<0.05$ for all genes between $T b k 1^{+/+} K I C$ and $T b k 1^{1 / 4} K I C$ tumors. (B) Representative images of tumors from Tbk $1^{1^{++}} \mathrm{KIC}$ and $T b k 1^{1 / 4} \mathrm{KIC}$ mice stained with E-cadherin, CK-19, claudin-1, Ki67, Slug, and Zeb-1. Images were taken at original magnification of $\times 20$ and quantified by normalizing the percentage of DAB to percentage area of tumor; $n \geq 4$ mice/ group. Results are representative of mean \pm SEM. ${ }^{*} P<0.05 ;{ }^{*} P<0.01$ by Mann-Whitney test.

TBK1 is central to Axl-driven epithelial plasticity. Pancreatic tumor cells frequently exploit EMT programs during metastatic dissemination $(23,26)$. However, the absence of functional TBK1 in pancreatic tumor cells limits EMT, invasion, and metastases. We recently reported that TBK1 is downstream of the receptor tyrosine kinase Axl, a receptor associated with $\operatorname{EMT}$ in $\operatorname{PDA}(27,28)$. Pharmacological inhibition of Axl led to a concentration-dependent decrease of TBK1 activity while the stimulation of Axl with its ligand, Gas6, resulted in TBK1 activation (28). To determine if TBK1 is central to Axl-driven EMT, we evaluated Axl signaling in $T b k 1^{1 / 4} K I C$ tumor cells. Axl was stimulated in Tbk $1^{4 / 4} K I C$ and $T b k 1^{+/+} K I C$ cells with AF854, an activating anti-Axl antibody (29), and the resulting cell lysates were probed for epithelial (E-cadherin, claudin-1), mesenchymal (N-cadherin, Slug), and Axl signaling targets (AKT). Axl activation induced N-cadherin and Slug protein by 2- to 3-fold in $\mathrm{Tbk}^{+/+} \mathrm{KIC}$ tumor cells while having no effect on mesenchymal markers in $\mathrm{Tbk} 1^{{ }^{\prime}}$ ${ }^{4} K I C$ tumor cells (Figure 8A). Furthermore, p-AKT levels increased 5-fold in $T b k 1^{+/+} K I C$ cells and remained

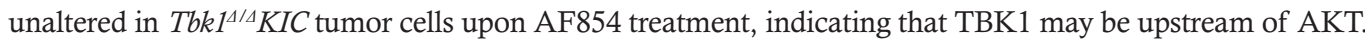
Next, we investigated Axl-induced RAS activation in $T b k 1^{+/+} K I C$ cell lines as a possible link between the Axl/ TBK1 signaling cascade. $T b k 1^{+/+} K I C$ - $A$ cells treated with AF854 showed a substantial increase of GTP-bound RAS, demonstrating that RAS activity is augmented by Axl activation (Figure 8B). These results were confirmed by using GTP and GDP loading as positive and negative controls, respectively (Supplemental Figure 7A). The activation of RAS with AF854 was also confirmed by inhibiting Axl, using BGB324, an established inhibitor of Axl activity (ref. 28 and Supplemental Figure 7B). Previous work has shown a RalB GTPase-mediated activation of TBK1 (12); therefore, we tested whether Axl-induced activation of RAS could increase GTP-bound RalB, mediating the activation of TBK1. Tbk1+/+KIC-A cells treated with AF854 showed a 3-fold increase in RalB GTP, demonstrating that RalB activity is increased upon Axl activation (Figure 8B). Axl-induced Ras and RalB activation was validated in an additional murine PDA cell line, KPfC-8, derived from 
A
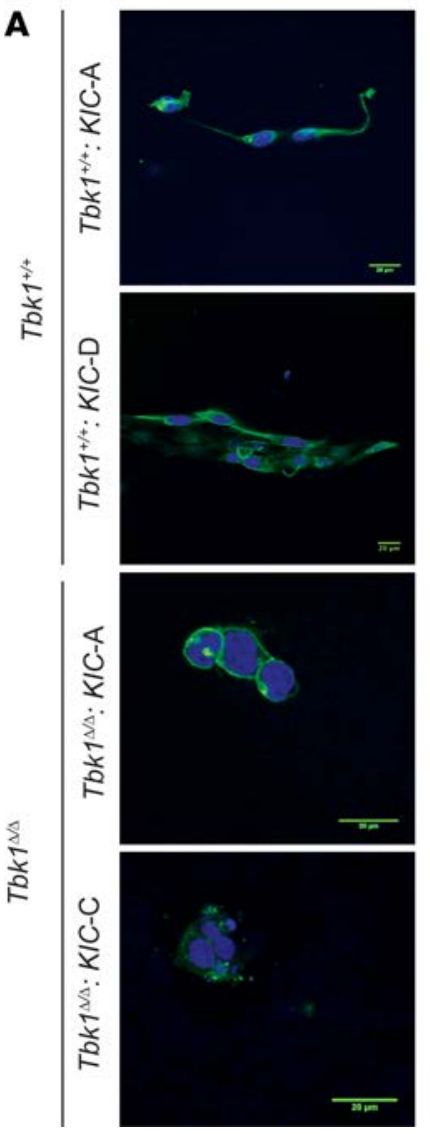

B

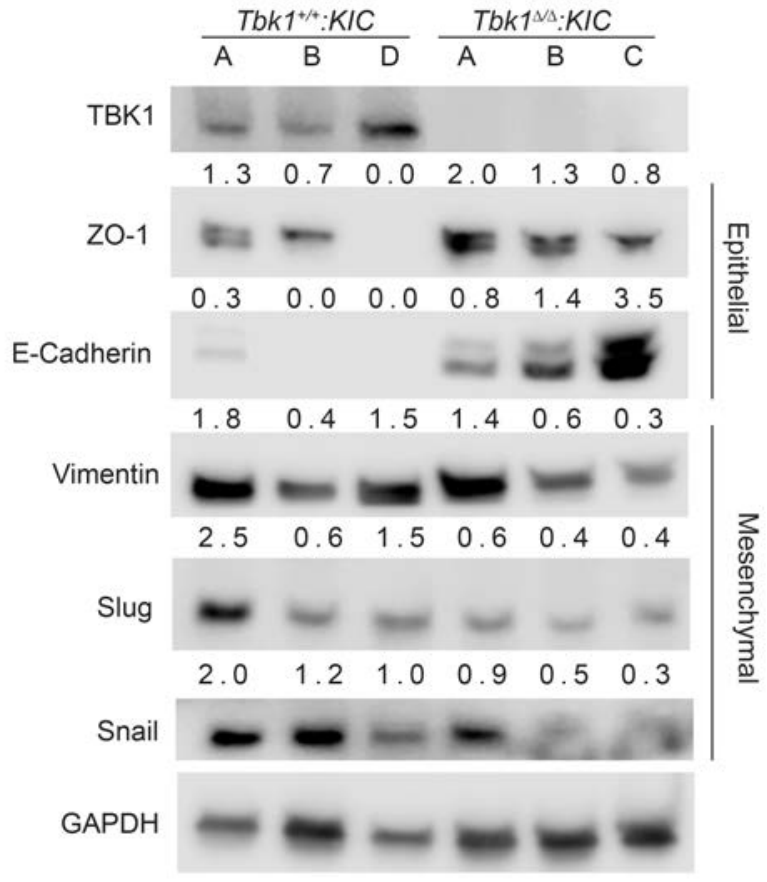

Figure 4. Tbk1 promotes pancreatic cancer epithelial plasticity. (A) Representative confocal images of single-cell

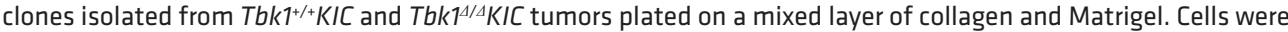
fixed and nuclei were labeled with DAPI (shown in blue); F-actin was labeled with phalloidin (green). Scale bars: 20 $\mu \mathrm{m}$. (B) Protein lysates isolated from $T b k 1^{1^{+/} K I C}$ and $T b k 1^{1 / 4} K I C$ cell lines were immunoblotted for indicated epithelial and mesenchymal markers. GAPDH was used as a loading control. Signal intensity of each sample was quantified and

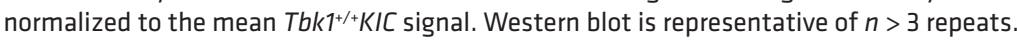

a spontaneous tumor in the Kras ${ }^{L L L-G 12 D /+}$ Trp $53^{\text {box } / \text { lox }} \mathrm{Ptfla}^{\text {Crel+ }}$ (KPfC) GEMM of PDA (Figure 8C). To extend and confirm these findings in human pancreatic cancer, we stimulated Panc-1 cells with Gas6, which we previously demonstrated increases TBK1 activity (28), and detected an increase in GTP-bound Ras and RalB (Figure 8D). These results are the first to our knowledge to show that Axl activates Ras and RalB, which leads to downstream activation of TBK1 and AKT, ultimately resulting in increased EMT and a more aggressive and metastatic tumor cell phenotype.

\section{Discussion}

Activating mutations in $K R A S$ are the dominant oncogenic drivers of pancreatic cancer $(3,4)$. No other common epithelial cancer has a single gene with comparable mutation frequency, yet efforts to therapeutically target mutant RAS proteins have not been successful (5). However, targeting signaling components downstream of RAS that are required for RAS-mediated oncogenesis presents a viable therapeutic alternative (30). TBK1 is a crucial effector of mutant active KRAS that we found to be expressed abundantly in $K R A S$-mutant PDA tumors and cell lines. Additionally, recent evidence suggests TBK1 expression correlates negatively with survival in human pancreatic cancer patients (11). The assessment of Tbk1 loss in multiple clinically relevant GEMMs of PDA revealed that mice with PDA lacking kinase-active TBK1 have substantially smaller and more epithelial tumors. Although survival was not significantly affected by the loss of Tbk1, Tbk1 loss resulted in fewer metastatic lesions relative to PDA mice with wild-type Tbk1. Mechanistic studies established that TBK1 promotes EMT downstream of Axl in PDA, providing insight into a novel function for TBK1. Further, these studies suggest that therapies targeting TBK1 could be used to exploit $K R A S$-mutant tumors. 
A

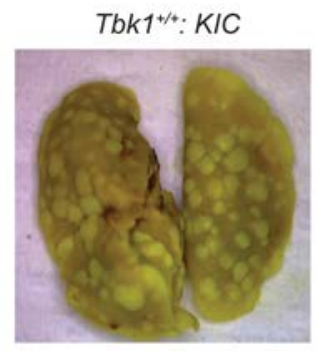

B

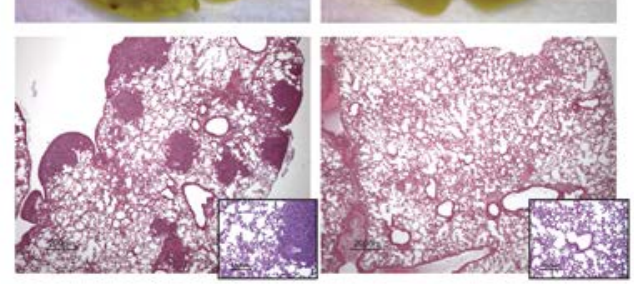

Tbk1 $1^{\mathrm{Na}}: K I C$

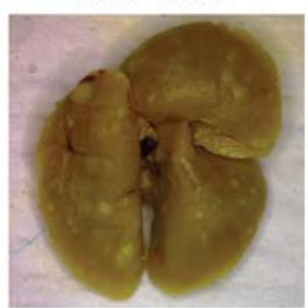

C

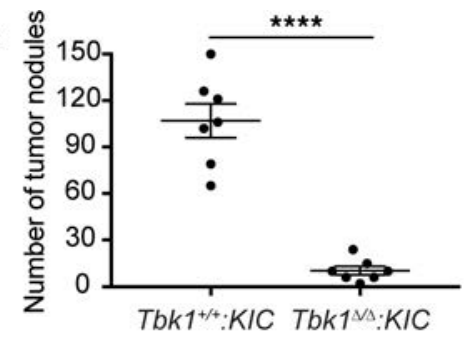

D

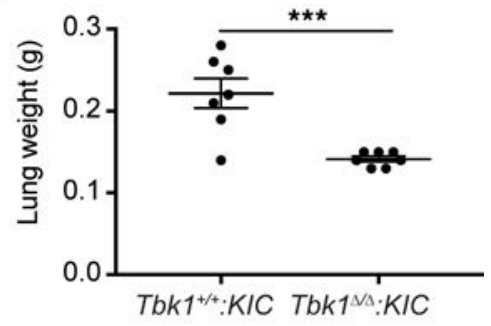

Figure 5. Loss of Tbk1 reduces lung colonization. (A) Representative images of lungs from NOD/SCID mice sacrificed 12 days after i.v. injection with $T b k 1^{+/+}$or $T b k 1^{1 / 4} K I C$ tumor cells (100,000 cells injected per mouse, $n=7$ mice/group). (B) H\&E sections of lungs from $\mathbf{A}$, highlighting the tumor nodules. Original magnification, $\times 4$ and $\times 20$ (insets). (C) Number of tumor nodules from $\mathbf{A}$. (D) Lung weights from $\mathbf{A}$. Results are representative of mean \pm SEM. ${ }^{* * *} P<0.001 ;{ }^{* * *} P<$ 0.0001 by unpaired, 2 -tailed $t$ test.

It is estimated that nearly $90 \%$ of cancer mortalities are due to metastases (31), yet TBK1 studies to date have evaluated the effect of TBK1 inhibition only on primary tumor burden. EMT is a hallmark of metastasis in pancreatic cancer and is critical to cancer cell dissemination (32-34). Within this morphological cellular program, epithelial cancer cells lose contact with the basement membrane and neighboring cells while gaining a more mesenchymal and invasive phenotype $(32,35)$. Our results show that tumors and isogenic cell lines from multiple pancreatic GEMMs that lack functional TBK1 are more epithelial in gene expression and morphology than PDA GEMM tumors containing wild-type Tbk1. These findings, in combination with mechanistic studies demonstrating that TBK1 is downstream of the EMT driver Axl, indicate that EMT in pancreatic tumor cells is halted by Tbk1 loss. TBK1 has been linked to EMT in other cancer types. In contrast with our results, knockdown of TBK1 in estrogen receptor- $\alpha$-positive (ER- $\alpha$-positive) breast cancer cells reportedly induced EMT and enhanced tumor growth and lung metastasis by suppressing ER $\alpha$ expression (13). However, in 2 recent studies, gene expression analysis revealed that a mesenchymal gene signature in melanoma and non-small cell lung cancer (NSCLC) cell lines was associated with sensitivity to TBK1 inhibition (TBK1i) $(36,37)$. Further analysis revealed mutations in $R A S$ family members as a common feature of NSCLC cell lines that showed sensitivity to TBK1i while NSCLC cells that were resistant to TBK1i had a more epithelial gene expression profile and less frequent activating RAS mutations (36). The mesenchymal gene signature in TBK1i-sensitive NSCLC lines is consistent with our observations in KRAS-driven $T b k 1^{+/+}$ KIC tumors that have undergone EMT. Moreover, the epithelial gene expression profile of TBK1-resistant NSCLC cell lines matches the epithelial phenotype of $T b k 1^{\Delta / \Delta} K I C$ tumors that grew independent of TBK1. Though the precise mechanism of how TBK1 promotes EMT is unclear, TBK1 can directly activate AKT (9). AKT activation can drive EMT via the induction of Snail and Slug that transcriptionally repress E-cadherin and induce vimentin, Twist1, MMP-2, and MMP-9, which promote tumor cell invasion $(35,38,39)$. Current studies are focused on understanding the interaction between TBK1 and AKT driving the mesenchymal phenotype in PDA and the identification of additional TBK1 substrates that promote EMT programs.

Interestingly, Tbk1-mutant KPC tumors were smaller than Tbk1 wild-type tumors, indicating that $T b k 1$ loss affects primary tumor growth in addition to tumor cell motility. In the KIC GEMMs, endpoint tumor

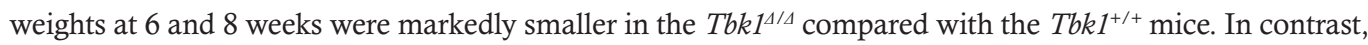
at 10 weeks there was no difference in endpoint tumor weights. Our results suggest $T b k 1^{\Delta / \Delta}$ tumor cells proliferate more quickly than $T b k 1^{+/+}$tumor cells as evidenced by in vitro cell proliferation assays and Ki67

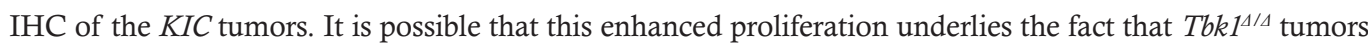
eventually catch up to the $T b k 1^{+/+}$tumors, in terms of tumor weight. 
A

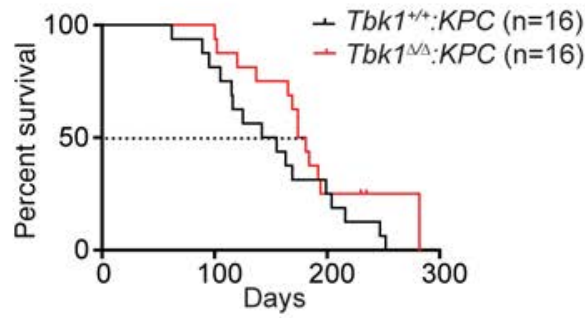

C

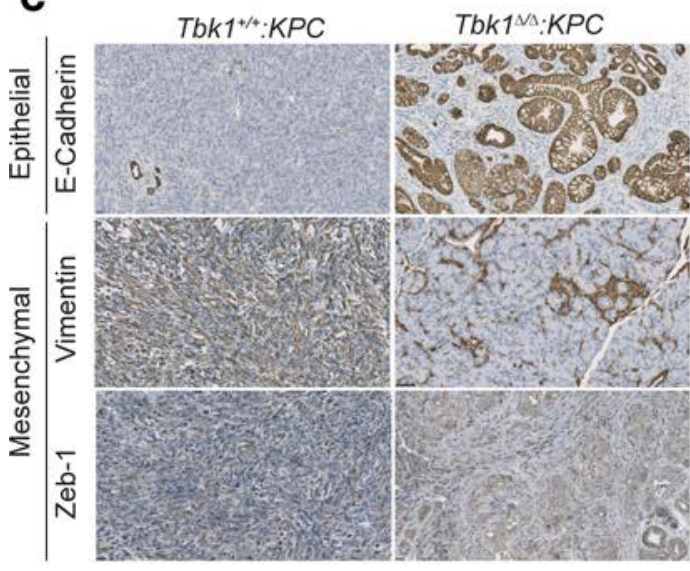

B

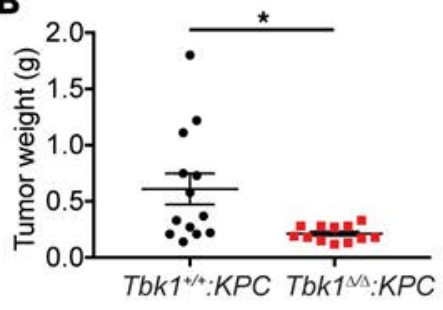

D

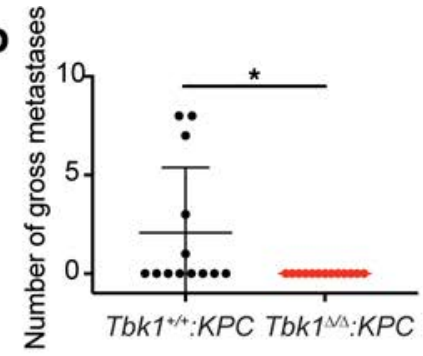

$\mathbf{E}$

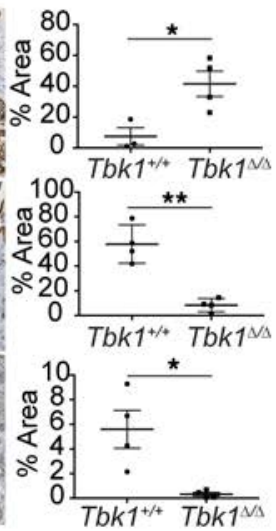

Tbk1 ${ }^{\text {Na }}: K P C$-Liver

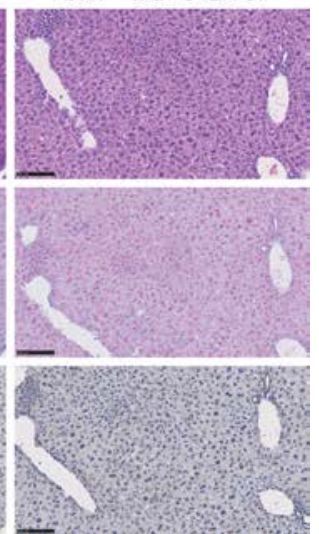

Figure 6. Loss of functional Tbk1 reduces metastasis. (A) Kaplan-Meier survival curve of $T b k 1^{1 / 4} K P C$ and $T b k 1^{+/+} K P C$ mice ( $n=16$ mice $\left./ g r o u p\right)$. A logrank Mantel-Cox test was used for survival comparison, $P=0.15$. (B) Tumor weights of $T b k{ }^{1 / / 4} K P C$ and $T b k 1^{+/+} K P C$ mice. (C) Representative images from tumors stained for E-cadherin, vimentin, and Zeb-1 from $T b k 1^{1 / 4} K P C$ and $T b k 1^{+/+} K P C$ mice. Original magnification, $\times 20$. Images were quantified by percentage of DAB divided by percentage hematoxylin area; $n \geq 4$ mice/group. (D) Number of gross metastases in $T b k 1^{1 / 4} K P C$ and $T b k 1^{+/+} K P C$ mice ( $n=13$ mice/group). (E) Representative liver histology in $T b k 1^{1 / 4} K P C$ and $T b k 1^{1^{+/}} K P C$ mice, including H\&E, Alcian blue, and CK-19 immunohistochemical staining. Scale bar: $100 \mu \mathrm{m}$. Original magnification, $\times 20 .{ }^{*} P<0.05 ;{ }^{*} P<0.01$ by unpaired, 2-tailed $t$ test.

TBK1 is central to numerous biological processes that could affect the growth of the primary tumor, including cell division, autophagy, innate immune response, and AKT/mTOR signaling (11, $36,37,40-45)$. In the context of pancreatic cancer, TBK1 has been reported to promote basal levels of autophagy as a means of silencing cytokine production (44). These findings imply that the inhibition or loss of TBK1 in PDA could increase cytokine production, ultimately driving immune activation and potentially an antitumor immune response. As previously mentioned, gene expression analysis revealed that $T b k 1^{4 / 4} K I C$ tumors displayed a higher expression of proinflammatory cytokines, including Cxcl1, Ccl2, Ccl4, Ccl27, Irf1, and Il1b. Although these results are not sufficient to conclude that $T b k 1$ loss promotes antitumor immunity in PDA, the idea is not unreasonable given that $T b k 1^{\Delta / \Delta}$ mice have been shown to produce higher levels of proinflammatory cytokines in response to immune challenge (17). In agreement with the notion that Tbk1 loss produces antitumor immunity, 2 recent studies reported that immune evasion and metastatic behavior are highly associated with the engagement of the cGAS/STING/TBK1 innate immune pathway in cancer cells (46-48). In the first study, Backhoum et al. (47) found that chromosomal instability in cancer cells, caused by errors in chromosomal segregation during mitosis, promoted cellular invasion and metastasis through the introduction of double-stranded DNA into the cytosol, engaging the cGAS/STING/TBK1 antiviral pathway. In the second report, Cañadas et al. (48) characterized an interferon-stimulated positive feedback loop of antisense endogenous retroviruses (ERVs) present in a number of human cancer cell lines that produced hyperactive innate immune signaling, myeloid cell infiltration, and immune checkpoint activation. Additionally, they discovered that high-ERV-expressing cancer cells correlate with an $\mathrm{Axl}^{+}$ mesenchymal state, which is consistent with our observations (48).

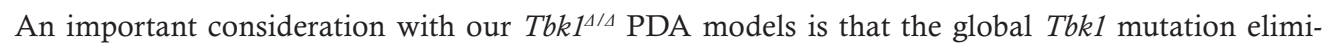
nates TBK1 activity and significantly reduces Tbk1 expression in all cell types, including immune cells, which could affect immune responses to tumor challenge. In fact, a recent study demonstrated that den- 
A
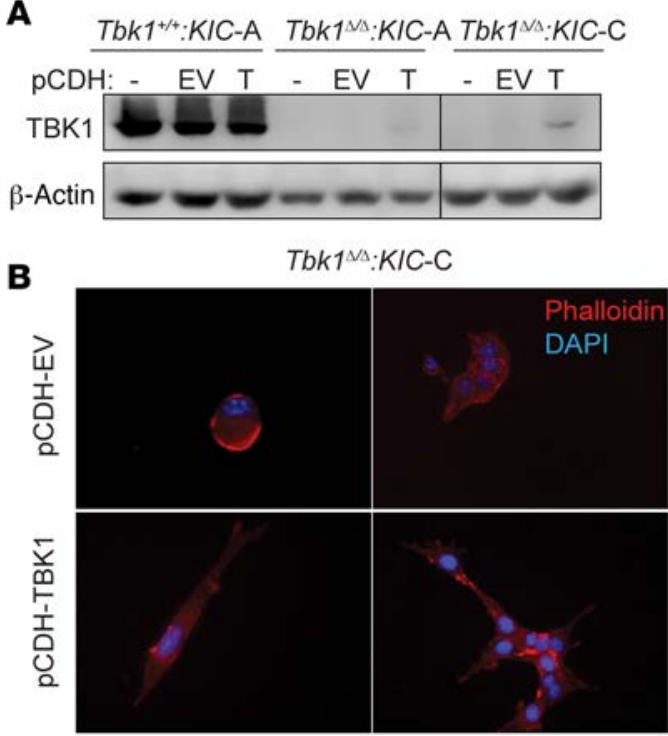

C
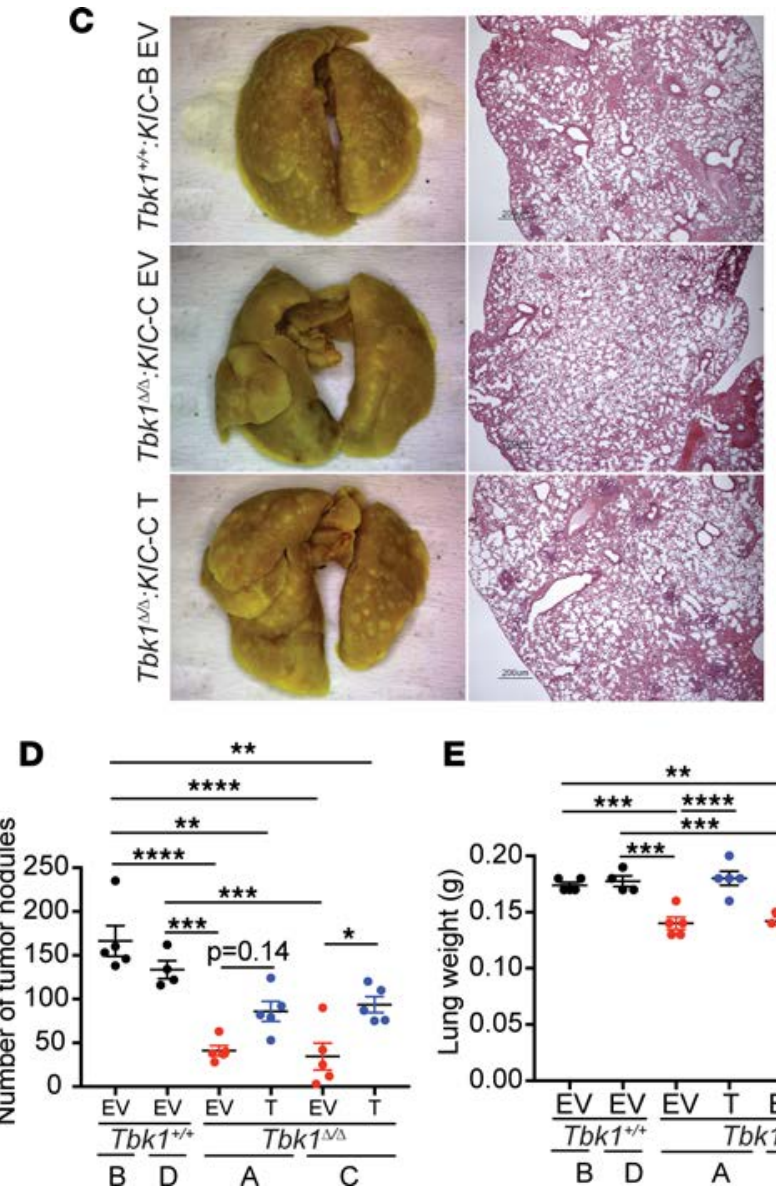

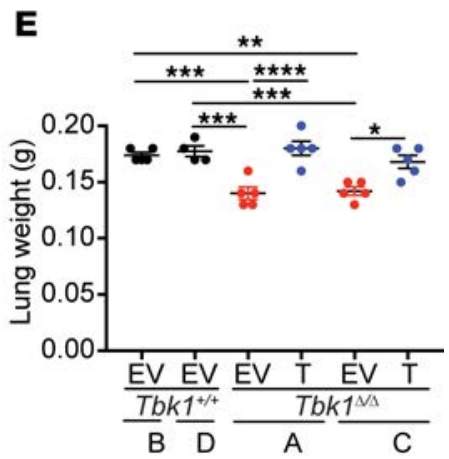

Figure 7. Reexpression of Tbk1 partially reverses colonization deficit in Tbk $\mathbf{1}^{1 / \Delta} \mathrm{KIC}$ cells. (A) Protein lysates from $\mathrm{Tbk} \mathbf{1}^{1 / \Delta} \mathrm{KIC}$ and $\mathrm{Tbk} \mathrm{T}^{+/+} \mathrm{KIC}$ cell lines infected with pCDH-empty vector (EV) or pCDH-TBK1 (T) were immunoblotted for TBK1. We used $\beta$-actin as a loading control. Solid line indicates where blot was cropped; however, all samples were run on the same gel and exposed simultaneously. (B) Representative images of $T b k 1^{1 / 4} K I C-C$ cells infected with EV or T plated on a mixed layer of collagen and Matrigel. Cells were fixed and nuclei were labeled with DAPI (show in blue); F-actin was labeled with phalloidin (red). (C) Representative images of lungs and H\&E (original magnification, $\times 20$ ) from NOD/SCID mice sacrificed 7 days after i.v. injection with Tbk1KIC EV and T cell lines (100,000 cells injected per mouse, $n=4-5$ mice/group). (D) Number of tumor nodules from C. (E) Lung weights from C. Results are representative of mean \pm SEM. ${ }^{*} P<0.05 ;{ }^{* *} P<0.01 ;{ }^{* *} P<0.001 ;{ }^{* * *} P<0.0001$ by 1 -way ANOVA with Tukey's multiple-comparisons test.

dritic cell-conditional Tbk1-knockout mice (Tbk1-DKO) injected subcutaneously with B16 melanoma cells lived longer and had smaller tumors compared with wild-type Tbk1 control mice (42). An assessment of B16 melanoma tumors from Tbk1-DKO animals revealed enhanced interferon-responsive gene expression and greater $\mathrm{T}$ effector cell infiltration into tumors and lymph nodes, confirming antitumor immunity conferred by dendritic cell Tbk1 loss. Collectively, these observations support a protumor immune function for TBK1 that could contribute to the larger tumor sizes in Tbk1 wild-type PDA mice.

Going forward, it will be important to understand the unique function of TBK1 in each relevant cell type within a tumor. Here we provide evidence of the effects of global Tbkl loss in all cell types with our $T b k 1^{4 / 4}$ PDA models, which in many respects is biologically analogous to the effects of pharmacologically inhibiting TBK1 systemically. Overall, our findings expand the spectrum of biological activities of TBK1 and suggest that the therapeutic inhibition of TBK1 may be a useful strategy to control tumor cell invasion and resulting metastases in RAS-driven cancers.

\section{Methods}

Animals. NOD/SCID mice were purchased from the University of Texas Southwestern Mouse Breeding Core. $T b k 1^{1 / 4}, \mathrm{Tbk1}^{+/+}, \mathrm{Kras}^{L S L-G 12 D /+} \mathrm{Cdkn} 2 a^{L o x / L o x}(\mathrm{KI})$, and Cdkn2a ${ }^{L o x / L o x} \mathrm{Ptfla}^{\mathrm{Cre} /+}$ (IC) mice were generated as pre-

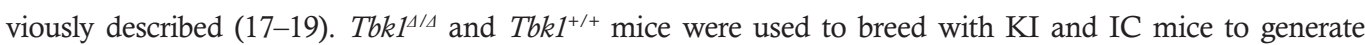

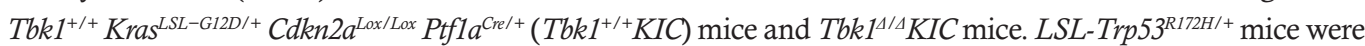




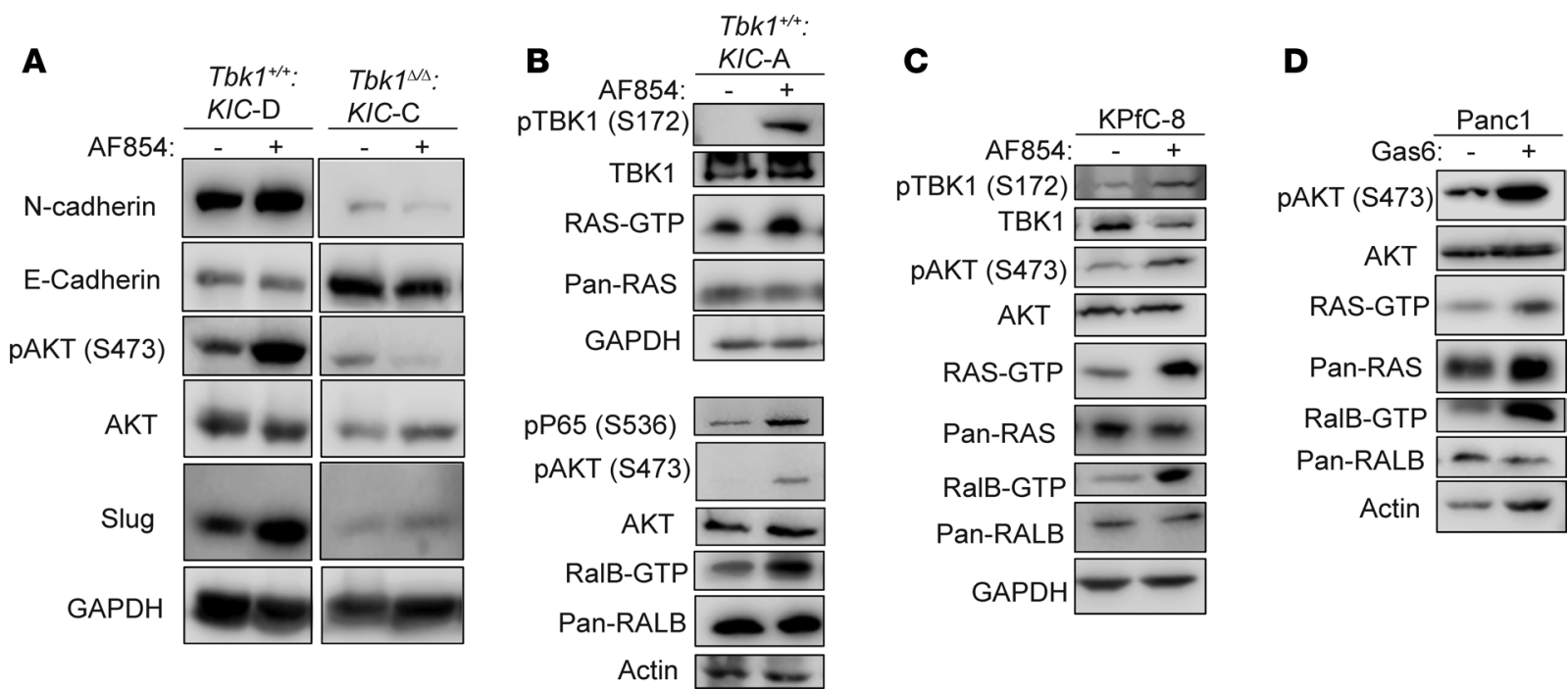

Figure 8. Tbk1 promotes epithelial plasticity downstream of AxI. (A) Protein lysates from $T b k{ }^{1++}{ }^{+} K I C-A$ cell lines treated with PBS or AF854 (6 nM) for 30 minutes were immunoblotted for indicated proteins. (B) Protein lysates from Tbk1KIC-A cells treated with PBS or AF854 (6 nM) for 30 minutes were assayed for active Ras and active RalB and immunoblotted for indicated proteins. (C) Protein lysates isolated from KPfC-8 cells treated with PBS or AF854 $(6 \mathrm{nM})$ for 30 minutes were assayed for active Ras and active RalB and immunoblotted for indicated proteins. (D) Protein lysates from Panc- 1 cells treated with PBS or $200 \mathrm{ng} / \mathrm{ml}$ Gas6 for 30 minutes were assayed for active Ras and active RalB and immunoblotted for indicated proteins. GAPDH and actin were used as loading controls. Lysates were run on parallel gels simultaneously and probed for phosphorylated and total proteins. Western blots displayed are representative of $n>3$ repeats for each activation assay.

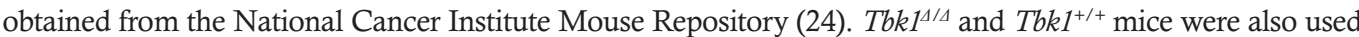

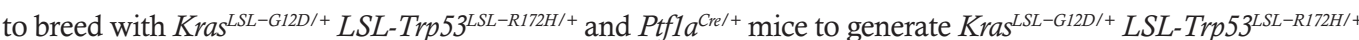
$P_{t f 1 a^{C r e /+}}(K P C)$ mice and $T b k 1^{4 / 4} K P C$ mice. All mice were bred and maintained in a pathogen-free barrier facility with access to food and water ad libitum.

Animal studies. All experiments were conducted using littermate-controlled mice. All mice were fed a normal chow diet (16\% protein diet, irradiated; Teklad Global Diets, Envigo). For endpoint studies, $T b k 1^{+/+} K I C$ and $T b k I^{\Delta / \Delta} K I C$ mice were sacrificed, and entire tissues, including pancreas/tumor, liver, lungs, and spleen, were harvested and weighed at 6,8 , and 10 weeks old, with $n=5-11$ mice per time point per group. $T b k 1^{+/+} K P C$ and $T b k 1^{\Delta / \Delta} K P C$ mice were sacrificed between 4 and 5 months, with $n \geq$ 8 mice per time point per group. For all survival studies, mice were carefully monitored and sacrificed when they appeared moribund. For lung colonization studies, Tbk $1^{+/+} K I C$ and $T b k 1^{\Delta / \Delta} K I C$ cells $\left(1 \times 10^{5}\right)$ were resuspended in $200 \mu \mathrm{lBS}$ and injected i.v. into the tail vein of 8-week-old female NOD/SCID mice. Lungs were harvested at 7 or 12 days after injection and fixed in Bouin's fixative (Polysciences, Inc.) for gross analysis of tumor nodules. Tumor colonization was analyzed by H\&E.

Histology and IHC. Pancreas/tumors, livers, lungs, spleens, and kidneys were excised and fixed with $10 \%$ neutral buffered formalin solution for 48 hours on a shaker at room temperature. Tissues were then washed and stored in PBS at $4^{\circ} \mathrm{C}$ and embedded in paraffin for sectioning by the UT Southwestern Molecular Pathology Core. All tissues were sectioned at $5 \mu \mathrm{m}$. After sectioning, slides were deparaffinized with xylene and rehydrated in a decreasing ethanol dilution series and then stained with H\&E, Masson's trichrome, or Alcian blue or fixed in 10\% neutral buffered formalin for 30 minutes before antigen retrieval. Sections for immunohistochemical fluorescence analysis were blocked with 5\% BSA and incubated with rabbit anti-vimentin (Cell Signaling Technology, 5741) in blocking solution (5\% BSA in TBS with $0.05 \%$ Tween) at $4^{\circ} \mathrm{C}$ overnight. TRITC-conjugated donkey anti-rabbit IgG (Jackson ImmunoResearch) was used as a secondary antibody. Sections for immunohistochemical DAB analysis were stained as previously described (49). Briefly, antigen retrieval was performed using antigen retrieval buffer $(10 \mathrm{mM}$ Tris- $\mathrm{HCl}$ and $1 \mathrm{mM}$ EDTA with $10 \%$ glycerol, $\mathrm{pH} 9$ ) heated at $110^{\circ} \mathrm{C}$ for 18 minutes. Sections were blocked with $2.5 \%$ goat serum (Vector Laboratories) for 30 minutes and incubated with anti-CK-19 (Abcam, ab15463), anti-E-cadherin (Cell Signaling Technology, 3195), anti-claudin-1 (Cell Signaling Technology, 13255), anti-Ki67 (Abcam, 15580), anti-Slug (Cell Signaling Technology, 9585), anti-Zeb-1 (Cell Signaling Technology, 3396), or anti-vimentin (Cell Signaling Technology, 5741) in this blocking solution ( $2.5 \%$ goat serum) at $4^{\circ} \mathrm{C}$ overnight. 
HRP-conjugated secondary anti-rabbit (ImmPRESS, Vector Laboratories) was used as secondary antibody. For chromogenic detection, sections were developed using Betazoid DAB (Biocare Medical, BDB2004L). After development, slides were counterstained with hematoxylin. Slides were mounted and coverslipped using VectaMount (Vector Laboratories, H-5501). Negative controls included omission of primary antibody. All slides were visualized at original magnification of $\times 20$ using Hamamatsu Nanozoomer 2.0-HT. Image analysis was conducted using Fiji software (https://imagej.net/Fiji) as previously described (49).

$R N A$ isolation and microarray analysis. Tumor tissues were excised from 8-week-old $T b k 1^{\Delta / \Delta}$ and $T b k 1^{+/+}$ KIC mice and snap-frozen with liquid nitrogen ( $n=3$ tumors per genotype). Total RNA was isolated after tissue homogenization in TRIzol (Thermo Fisher Scientific), and RNA was extracted using an RNeasy RNA extraction kit (Qiagen). RNA was quantified using a NanoDrop instrument (Thermo Fisher Scientific) and checked for quality with a Bioanalyzer instrument (Agilent). Gene expression was analyzed on a MouseWG-6 v2.0 Expression BeadChip (Illumina) through the UT Southwestern Microarray Core. Gene expression data analysis was performed through IPA software (Qiagen, www.qiagenbioinformatics.com/ products/ingenuity-pathway-analysis/). Java TreeView (http://jtreeview.sourceforge.net/) and Cluster 3.0 software (http://bonsai.hgc.jp/ mdehoon/software/cluster/software.htm) were used for hierarchical clustering gene expression analysis (50). Data was deposited in Gene Expression Omnibus (https://www. ncbi.nlm.nih.gov/geo/query/acc.cgi?acc=GSE130232) with the accession number CSE130232.

Recombined Cdkn2a allele detection. Liver micrometastasis was assessed by quantitative reverse transcription PCR (RT-PCR) for the recombined Cdkn2a(Ink4a/Arf) allele. Briefly, frozen livers were homogenized in SDS lysis buffer (100 mM Tris, $\mathrm{pH} 8.8 ; 5 \mathrm{mM}$ EDTA, $0.2 \%$ SDS, $100 \mathrm{mM} \mathrm{NaCl}$ ) and digested at $56^{\circ} \mathrm{C}$ overnight. DNA was extracted using phenol/chloroform/isoamyl alcohol (25:24:1), and quantitative RT-PCR was performed using iQ SYBR Green Supermix (Bio-Rad). The following validated primers were used for analysis of Cdkn2a: GCCGACATCTCTCTGACCTC (forward) and CTCGAACCAGGTTTCCATTG (reverse). Each sample was analyzed in triplicate.

Immunoblotting. Tissues and cells were lysed in ice-cold RIPA buffer (50 mM Tris-Cl, $150 \mathrm{mM}$ $\mathrm{NaCl}, 1 \%$ Nonidet P-40, $0.5 \%$ sodium deoxycholate, and $0.1 \%$ SDS) containing cocktails of protease (Thermo Fisher Scientific) and phosphatase inhibitors (MilliporeSigma) and centrifuged for 20 minutes at $13,000 \mathrm{~g}$ at $4^{\circ} \mathrm{C}$. Total protein concentration was calculated using a bicinchoninic acid assay kit (Thermo Fisher Scientific). Proteins were resolved by SDS-PAGE and transferred to a methanol-activated polyvinylidene difluoride membrane. All primary and secondary antibodies were diluted in $5 \%$ donkey serum in TBS with $0.05 \%$ Tween. Primary antibodies used included the following: anti-p-AKT (S473, Cell Signaling Technology, 4060), anti-AKT (Cell Signaling Technology, 9272), anti-p-Axl (Y779, R\&D, AF2228), anti-Axl (Santa Cruz Biotechnology, sc-1096), anti-claudin-1 (Cell Signaling Technology, 13255), anti-E-cadherin (Cell Signaling Technology, 3195), anti-IRF3 (Santa Cruz Biotechnology, sc-9082), anti-N-cadherin (Cell Signaling Technology, 13116), anti-pp65 (Cell Signaling Technology, 3033), anti-Ras (Abcam, ab108602), anti-Snail (Cell Signaling Technology, 3879), anti-Slug (Cell Signaling Technology, 9585), anti-p-TBK1 (S172, Cell Signaling Technology, 5483, and Abcam, 109272), anti-TBK1 (Abcam, ab40676), anti-vimentin (Cell Signaling Technology, 5741), anti-Zeb-1 (Cell Signaling Technology, 3396), and anti-ZO-1 (Cell Signaling Technology, 8193). Anti- $\beta$-actin (MilliporeSigma, A2066) and anti-GAPDH (Cell Signaling Technology, 2118) were used as loading controls for all Western blots shown. HRP-conjugated donkey anti-rabbit, donkey anti-mouse, and donkey anti-goat IgG (1:10,000, Jackson ImmunoResearch) were used as secondary antibodies. Membranes were exposed with Clarity Western ECL Blotting Substrate (Bio-Rad) and visualized with the Odyssey Fc imager (LI-COR Biotechnology). Each Western blot was repeated at least 3 times; representative experiments are displayed.

Cell lines. Human and mouse cancer cell lines (AsPC-1, Capan-1, Hs766T, MCF7, MIA PaCa-2, PANC-1, PL-45) were obtained from ATCC. HPNEs were generated as previously described (16) and obtained from the UT MD Anderson Cancer Center. The KPC-M09 and KPfC-8 cell lines were isolated from spontaneous tumors originating in a KPC or KPfC $\left(\mathrm{Kras}^{L S L-G 12 D /+} \operatorname{Trp} 53^{\text {lox } / l o x} \mathrm{Ptfla} \mathrm{a}^{\mathrm{Cre} /+}\right)$ mouse, respectively, as previously described (28). All cell lines were cultured in DMEM or RPMI medium (Invitrogen) containing $10 \%$ FBS and $1 \times$ penicillin/streptomycin and maintained in a humidified incubator with $5 \%$ $\mathrm{CO}_{2}$ at $37^{\circ} \mathrm{C}$. The human cell lines were DNA fingerprinted for provenance using the Power-Plex $1.2 \mathrm{kit}$ (Promega) and confirmed to be the same as the DNA fingerprint library maintained by ATCC. All cell lines were confirmed to be free of Mycoplasma (e-Myco kit, Boca Scientific) before use. 
Isogenic cell lines were derived from individual tumors of 8 -week-old $T b k 1^{+/+} K I C$ and $T b k 1^{\Delta / \Delta} K I C$ mice and 3- to 5-month-old $T b k 1^{+/+} K P C$ and $T b k 1^{\Delta / \Delta} K P C$ mice. Each tumor was minced and digested with $1 \%$ collagenase type I, DMEM, $10 \mathrm{mM}$ HEPES, and $1 \% \mathrm{FBS}$ at $37^{\circ} \mathrm{C}$ to obtain a single-cell suspension. Cell suspensions were centrifuged at low speed $(1,000 \mathrm{x} g)$ to pelletize large debris, resuspended in wash buffer, and passed through a $70-\mu \mathrm{m}$ cell strainer. The resulting cell suspension was plated at low density to isolate tumor cell populations using cloning rings (Scienceware). Cells were confirmed to be tumor cells by immunocytochemistry and PCR. These cell lines were expanded and stained for tumor cell markers. Cell lines were confirmed to be pathogen free before

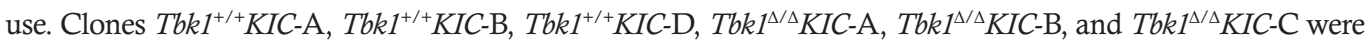
used in subsequent experiments, as well as $T b k 1^{+/+} K P C$ and $T b k 1^{\Delta / \Delta} K P C$ cell lines. Cells were cultured in DMEM containing $10 \% \mathrm{FBS}$ and maintained at $37^{\circ} \mathrm{C}$ in a humidified incubator with $5 \% \mathrm{CO}_{2}$ and $95 \% \mathrm{O}_{2}$.

Wound healing and invasion assays. Wound healing assays were conducted in 6-well plates. Monolayers of cells were grown in low-serum medium until 90\% confluence was reached. Each well was scratched with a P200 pipette tip to create an artificial wound, washed with PBS to remove residual cells, and replaced with fresh medium containing 10\% FBS. Cells were photographed at indicated time points after wounding. Wound closure was measured as a percentage of original wound width with MRI Wound Healing Tool macro (ImageJ, NIH).

Invasion assays were carried out with QCM ECMatrix Cell Invasion Assays (MilliporeSigma). In brief, cells were serum-starved overnight and then seeded the next day on Transwell inserts (8- $\mu \mathrm{m}$ pore size) that were lined with a reconstituted basement membrane matrix of proteins derived from the Engelbreth-HolmSwarm mouse tumor. The inner chambers were filled with serum-free medium while the outer chambers were filled with medium containing $10 \%$ FBS as the chemoattractant. After the indicated time points, invaded cells on the bottom of the insert membrane were dissociated from the membrane when incubated with cell detachment buffer and subsequently lysed and detected by CyQUANT GR dye. Each experiment was repeated at least 3 times; representative experiments are displayed.

Organotypic culture and immunocytochemistry. For each cell line, 2000 cells were plated in 8-well chamber slides onto a base layer of growth factor-reduced Matrigel $(5 \mathrm{mg} / \mathrm{ml}, \mathrm{BD}$ Biosciences, Lot A6532) and collagen type I (1.5-2.1 mg/ml, BD Biosciences) and cultured for 3 to 4 days in a humidified $37^{\circ} \mathrm{C}$ incubator as previously described (51). For immunocytochemistry, cultures were fixed in 2\% formalin (MilliporeSigma) in PBS for 20 minutes, permeabilized with $0.5 \%$ Triton X-100 in PBS for 10 minutes at room temperature, incubated with Alexa Fluor 488 Phalloidin (A12379, Invitrogen) or Alexa Fluor 546 Phalloidin (A22283, Invitrogen) in immunofluorescence buffer as described (52) for 1 hour at room temperature, and mounted using ProLong Gold antifade reagent with DAPI (Invitrogen). Images were acquired using a confocal laser-scanning microscope (LSM880, Zeiss) through UT Southwestern Live Cell Imaging Facility (Dallas, Texas) and a Nikon Eclipse E600 microscope with a Nikon Digital Dx1200me camera.

Active GTPase assays. Active RAS in cell lysates was measured via precipitation with GST-tagged RAFRas-binding domain beads (Ras Pull-down Activation Assay Biochem Kit, Cytoskeleton, Inc.). Active RalB in cell lysates was measured via precipitation with RalBP1 PBD Agarose beads (Cell BioLabs, Inc.). Lysates were prepared and precipitation was performed per manufacturer instructions. Subsequently, pull-down samples and respective whole cell lysates were immunoblotted with anti-RAS (pan) (Abcam, catalog ab108602) or antiRALB (pan) (Abcam, catalog ab223479) and indicated loading controls.

Reagents. The mammalian expression plasmid pCDH-CMV-MCS-TBK1-EF1-NEO was provided by Peiqing Shi and James Chen (UT Southwestern Medical Center). Lentiviral-based expression constructs were packaged by cotransfection of HEK293T cells with psPAX2 and pMD2.G packaging system (4:2:1). Polyethylenimine was used for transfection at a 3:1 ratio of total DNA. Transfection medium was replaced with 10\% complete DMEM 24 hours after transfection and incubated a further 24 hours before viral particle collection. $T b k 1^{+/+}$and $T b k 1^{\Delta / 4} K I C$ cells were seeded at a density of $1 \times 10^{6}$ cells per $10-\mathrm{cm}$ dish. The cells were infected with lentiviral particles and polybrene $(10 \mu \mathrm{g} / \mathrm{ml}) 24$ hours later. At 24 hours after infection, cells were given fresh medium containing G418 (400 $\mu \mathrm{g} / \mathrm{ml}$, InvivoGen) for selection and maintained in culture under selection for 3 weeks following initial infection. AF854 (R\&D Systems) was previously shown to activate mouse Axl and was used at indicated concentrations to stimulate mouse Axl (29).

Statistics. Statistical analyses were performed using GraphPad Prism. All data are expressed as mean \pm SEM. All data were analyzed by unpaired, 2-tailed $t$ test, Mann-Whitney test, or ANOVA with Tukey's multiple comparison's test, with the exception of survival comparisons, which were analyzed using logrank Mantel-Cox tests. Statistical significance was accepted at $P<0.05$, with asterisks denoting $P$-value levels: ${ }^{*} P<0.05 ;{ }^{* *} P<0.01 ;{ }^{* * *} P<0.001$; and ${ }^{* * * *} P<0.0001$. 
Study approval. All experiments were approved by the UT Southwestern Medical Center Institutional Animal Care and Use Committee (Dallas, Texas, USA) and were performed in compliance with UT Southwestern institutional guidelines and with relevant laws.

\section{Author contributions}

VHC, ENA, and RAB conceived and designed the study. VHC, ENA, WD, and AEB acquired data and performed analysis and interpretation of data. VHC and ENA wrote the manuscript. RAB reviewed and revised the manuscript. RAB supervised the study.

\section{Acknowledgments}

We thank our UT Southwestern colleagues Jonathan Cooper and Aubishek Zaman for technical advice and shared resources, Peiqing Shi and James Chen for the TBK1 expression construct, and Dave Primm for editorial assistance. We also thank Tae Hyun Hwang for assistance with clinical analysis. We gratefully acknowledge Brekken lab members Jason Toombs, Tara Billman, Dan Ye, and Melissa Gross for their assistance with the mouse studies. The work was supported by NIH grants R01 CA192381 and U54 CA210181 Project 2 to RAB and T32 CA124334 (principal investigator: J. Shay, UT Southwestern, Dallas, Texas) to VHC and by the Effie Marie Cain Scholarship in Angiogenesis Research and the Gillson Longenbaugh Foundation to RAB. The funders had no role in study design, data collection and analysis, decision to publish, or preparation of the manuscript.

Address correspondence to: Rolf A. Brekken, Hamon Center for Therapeutic Oncology Research, University of Texas Southwestern Medical Center, 6000 Harry Hines Blvd., Dallas, Texas 75390-8593, USA. Phone: 214.648.5151; Email: rolf.brekken@utsouthwestern.edu.

1. Ferlay J, Shin HR, Bray F, Forman D, Mathers C, Parkin DM. Estimates of worldwide burden of cancer in 2008: GLOBOCAN 2008. Int J Cancer. 2010;127(12):2893-2917.

2. Hezel AF, Kimmelman AC, Stanger BZ, Bardeesy N, Depinho RA. Genetics and biology of pancreatic ductal adenocarcinoma. Genes Dev. 2006;20(10):1218-1249.

3. Hingorani SR, et al. Preinvasive and invasive ductal pancreatic cancer and its early detection in the mouse. Cancer Cell. 2003;4(6):437-450.

4. Karnoub AE, Weinberg RA. Ras oncogenes: split personalities. Nat Rev Mol Cell Biol. 2008;9(7):517-531.

5. Gysin S, Salt M, Young A, McCormick F. Therapeutic strategies for targeting ras proteins. Genes Cancer. 2011;2(3):359-372.

6. Neel NF, Martin TD, Stratford JK, Zand TP, Reiner DJ, Der CJ. The RalGEF-Ral effector signaling network: The road less traveled for anti-Ras drug discovery. Genes Cancer. 2011;2(3):275-287.

7. Lim KH, et al. Divergent roles for RalA and RalB in malignant growth of human pancreatic carcinoma cells. Curr Biol. 2006;16(24):2385-2394.

8. Lim KH, et al. Activation of RalA is critical for Ras-induced tumorigenesis of human cells. Cancer Cell. 2005;7(6):533-545.

9. Ou YH, et al. TBK1 directly engages Akt/PKB survival signaling to support oncogenic transformation. Mol Cell. 2011;41(4):458-470.

10. Barbie DA, et al. Systematic RNA interference reveals that oncogenic KRAS-driven cancers require TBK1. Nature. 2009;462(7269):108-112.

11. Cruz VH, Brekken RA. Assessment of TANK-binding kinase 1 as a therapeutic target in cancer. J Cell Commun Signal. 2018;12(1):83-90

12. Chien Y, et al. RalB GTPase-mediated activation of the IkappaB family kinase TBK1 couples innate immune signaling to tumor cell survival. Cell. 2006;127(1):157-170.

13. Yang KM, et al. Loss of TBK1 induces epithelial-mesenchymal transition in the breast cancer cells by ER $\alpha$ downregulation. Cancer Res. 2013;73(22):6679-6689.

14. Korherr C, et al. Identification of proangiogenic genes and pathways by high-throughput functional genomics: TBK1 and the IRF3 pathway. Proc Natl Acad Sci U S A. 2006;103(11):4240-4245.

15. Shen RR, Hahn WC. Emerging roles for the non-canonical IKKs in cancer. Oncogene. 2011;30(6):631-641.

16. Lee KM, Yasuda H, Hollingsworth MA, Ouellette MM. Notch 2-positive progenitors with the intrinsic ability to give rise to pancreatic ductal cells. Lab Invest. 2005;85(8):1003-1012.

17. Marchlik E, et al. Mice lacking Tbk1 activity exhibit immune cell infiltrates in multiple tissues and increased susceptibility to LPS-induced lethality. J Leukoc Biol. 2010;88(6):1171-1180.

18. Aguirre AJ, et al. Activated Kras and Ink4a/Arf deficiency cooperate to produce metastatic pancreatic ductal adenocarcinoma. Genes Dev. 2003;17(24):3112-3126.

19. Bardeesy N, DePinho RA. Pancreatic cancer biology and genetics. Nat Rev Cancer. 2002;2(12):897-909.

20. Aguilera KY, et al. Collagen signaling enhances tumor progression after anti-VEGF therapy in a murine model of pancreatic ductal adenocarcinoma. Cancer Res. 2014;74(4):1032-1044.

21. Zolghadri Y, et al. Malnutrition in pancreatic ductal adenocarcinoma (PDA): dietary pancreatic enzymes improve short-term health but stimulate tumor growth. Am J Pathol. 2018;188(3):616-626. 
22. Shintani Y, Fukumoto Y, Chaika N, Svoboda R, Wheelock MJ, Johnson KR. Collagen I-mediated up-regulation of N-cadherin requires cooperative signals from integrins and discoidin domain receptor 1. J Cell Biol. 2008;180(6):1277-1289.

23. Krebs AM, et al. The EMT-activator Zeb1 is a key factor for cell plasticity and promotes metastasis in pancreatic cancer. Nat Cell Biol. 2017;19(5):518-529.

24. Hingorani SR, et al. Trp53R172H and KrasG12D cooperate to promote chromosomal instability and widely metastatic pancreatic ductal adenocarcinoma in mice. Cancer Cell. 2005;7(5):469-483.

25. Wang M, et al. Fibulin-5 blocks microenvironmental ROS in pancreatic cancer. Cancer Res. 2015;75(23):5058-5069.

26. Ostapoff KT, et al. Neutralizing murine TGF $\beta$ R2 promotes a differentiated tumor cell phenotype and inhibits pancreatic cancer metastasis. Cancer Res. 2014;74(18):4996-5007.

27. Kirane A, et al. Warfarin blocks Gas6-mediated Axl activation required for pancreatic cancer epithelial plasticity and metastasis Cancer Res. 2015;75(18):3699-3705.

28. Ludwig KF, et al. Small-molecule inhibition of Axl targets tumor immune suppression and enhances chemotherapy in pancreatic cancer. Cancer Res. 2018;78(1):246-255.

29. Zagórska A, Través PG, Lew ED, Dransfield I, Lemke G. Diversification of TAM receptor tyrosine kinase function. Nat Immunol. 2014;15(10):920-928.

30. Engelman JA, et al. Effective use of PI3K and MEK inhibitors to treat mutant Kras G12D and PIK3CA H1047R murine lung cancers. Nat Med. 2008;14(12):1351-1356.

31. Weigelt B, Peterse JL, van 't Veer LJ. Breast cancer metastasis: markers and models. Nat Rev Cancer. 2005;5(8):591-602.

32. Puls TJ, Tan X, Whittington CF, Voytik-Harbin SL. 3D collagen fibrillar microstructure guides pancreatic cancer cell phenotype and serves as a critical design parameter for phenotypic models of EMT. PLoS One. 2017;12(11):e0188870.

33. Gaianigo N, Melisi D, Carbone C. EMT and treatment resistance in pancreatic cancer. Cancers (Basel). $2017 ; 9(9): \mathrm{E} 122$.

34. Wang S, Huang S, Sun YL. Epithelial-mesenchymal transition in pancreatic cancer: a review. Biomed Res Int. 2017;2017:2646148.

35. Larue L, Bellacosa A. Epithelial-mesenchymal transition in development and cancer: role of phosphatidylinositol 3' kinase/ AKT pathways. Oncogene. 2005;24(50):7443-7454.

36. Cooper JM, et al. TBK1 provides context-selective support of the activated AKT/mTOR pathway in lung cancer. Cancer Res. 2017;77(18):5077-5094.

37. Eskiocak B, et al. Biomarker accessible and chemically addressable mechanistic subtypes of BRAF melanoma. Cancer Discov. $2017 ; 7(8): 832-851$

38. Grille SJ, et al. The protein kinase Akt induces epithelial mesenchymal transition and promotes enhanced motility and invasiveness of squamous cell carcinoma lines. Cancer Res. 2003;63(9):2172-2178.

39. Xu W, Yang Z, Lu N. A new role for the PI3K/Akt signaling pathway in the epithelial-mesenchymal transition. Cell Adh Migr. 2015;9(4):317-324.

40. Pillai S, Nguyen J, Johnson J, Haura E, Coppola D, Chellappan S. Tank binding kinase 1 is a centrosome-associated kinase necessary for microtubule dynamics and mitosis. Nat Commun. 2015;6:10072.

41. Kim JY, et al. Dissection of TBK1 signaling via phosphoproteomics in lung cancer cells. Proc Natl Acad Sci U S A. 2013;110(30):12414-12419.

42. Xiao Y, et al. The kinase TBK1 functions in dendritic cells to regulate T cell homeostasis, autoimmunity, and antitumor immunity. J Exp Med. 2017;214(5):1493-1507.

43. Zhu Z, et al. Inhibition of KRAS-driven tumorigenicity by interruption of an autocrine cytokine circuit. Cancer Discov. 2014;4(4):452-465.

44. Yang S, et al. Autophagy inhibition dysregulates TBK1 signaling and promotes pancreatic inflammation. Cancer Immunol Res. 2016;4(6):520-530.

45. Helgason E, Phung QT, Dueber EC. Recent insights into the complexity of Tank-binding kinase 1 signaling networks: the emerging role of cellular localization in the activation and substrate specificity of TBK1. FEBS Lett. 2013;587(8):1230-1237.

46. Bakhoum SF, Cantley LC. The multifaceted role of chromosomal instability in cancer and its microenvironment. Cell. 2018;174(6):1347-1360

47. Bakhoum SF, et al. Chromosomal instability drives metastasis through a cytosolic DNA response. Nature. 2018;553(7689):467-472

48. Cañadas I, et al. Tumor innate immunity primed by specific interferon-stimulated endogenous retroviruses. Nat Med. 2018;24(8):1143-1150

49. Sorrelle $\mathrm{N}$, et al. Improved multiplex immunohistochemistry for immune microenvironment evaluation of mouse formalin-fixed, paraffin-embedded tissues. J Immunol. 2019;202(1):292-299.

50. Keil C, Leach RW, Faizaan SM, Bezawada S, Parsons L, Baryshnikova, A. Treeview 3.0 ( $\alpha 3$ ) — Visualization and analysis of large data matrices. Zenodo Web site. http://doi.org/10.5281/zenodo.160573. Published October 13, 2016. Accessed April 3, 2019.

51. Westcott JM, et al. An epigenetically distinct breast cancer cell subpopulation promotes collective invasion. J Clin Invest. 2015;125(5):1927-1943.

52. Pearson GW, Hunter T. Real-time imaging reveals that noninvasive mammary epithelial acini can contain motile cells. $J$ Cell Biol. 2007;179(7):1555-1567. 